\title{
LA EDUCACIÓN SUPERIOR DESDE LAS EXIGENCIAS DEL SIGLO XXI (Lineamientos Curriculares)
}

\author{
NeLson ERNesto LóPEZ JineNeZ *
}

\section{INTRODUCCIÓN}

Es preciso señalar que los componentes de este escrito pretenden retomar los dilemas que se presentan al analizar conceptos como disciplina, profesión, oficio, pero inscritos en un contexto socio económico, político y cultural lo más amplio posible, que saque la discusión del nivel semántico y la ubique en el campo de la reorganización y la transformación. muy en consonancia con las exigencias y retos planteados como consecuencia del advenimiento de un nuevo siglo y la necesidad de recomposición derivadas de la reorganización del capitalismo al final del siglo en donde "el sistema de mercado es mostrado como el único posible, el más justo y el que reconstruye las interacciones sociales bajo el pretexto de que no existe otro modelo de desarrollo posible". ${ }^{1}$

No obstante, hay que advertir que las diferentes posiciones son producto más de la incertidumbre que de la certeza, propiciando así el diálogo, el análisis y la reflexión en torno al rumbo que debe orientar el desarrollo de la Universidad, que al ser asumida como "organización social efectiva lo primero que debe asegurar es su supervivencia en un medio cambiante y para ello debe contar con la capacidad de adaptarse e integrarse creativamente a ese entorno y crear nuevas realidades, es decir, de aprender continuamente"z

En este planteamiento se inscribe la concepción del currículo como una acción eminentemente investigativa, producto del trabajo colectivo, al cual se accede por aproximaciones sucesivas; desestimulando los diferentes intentos de otorgarle una dimensión puramente instrumental y mecánica, en donde la labor del docente se reduce a un acto simple y llano de operacionalización.

Por ello en el desarrollo de los tópicos estructurantes de esta conferencia, se presentarán los argumentos necesarios para señalar el carácter de constructores de currículo que hoy debemos impulsar y lograr como respuesta a nuestra pretensión de intelectuales y trabajadores de la cultura, muy en consonancia, con la responsabilidad histórica que hemos asumido, cuando detectamos nuestra condición de formadores de proyectos de vida a través de acciones de profesionalización en las diferentes áreas o expresiones del conocimiento.

* Investigador principal Provecto Currículo y Calidad de la Educación Superior en Colombia (fase III). ICFESUNIVERSIDAD SURCOLUMBIANA - 1997.

1 Mejía, Marco Raúl. Reconstruyendo la Transformación Social, Movimientos Sociales y Educación Popular. Mesa redonda. Magisterio. Agosto-1966.

2 Colombia al Filo de la Oportunidad. Misión Ciencia, Educación y Desarrollo 1995. 
1. ELEMENTOS ESTRUCTURANTES DEL CONTEXTO SOCIO-ECONÓMICO, POLÍTICO Y CULTURAL AD.PORTAS DEL SIGLO XXI.

En un intento por contextualizar la problemática curricular en la realidad actual, resulta de suma importancia destacar algunos de los elementos que nos indican que hoy estamos en una nueva época: la del conocimiento y el desarrollo tecnológico en el campo de la informatización y su aplicación en la vida cotidiana, así, lo digital, propiciador de la informática, de la imagen, etc., se convierte en la tecnología intelectual dominante, razón por la cual es conveniente señalar algunos de los rasgos característicos del modelo prevaleciente:

- Poca capacidad de

D - Cambios en los me-

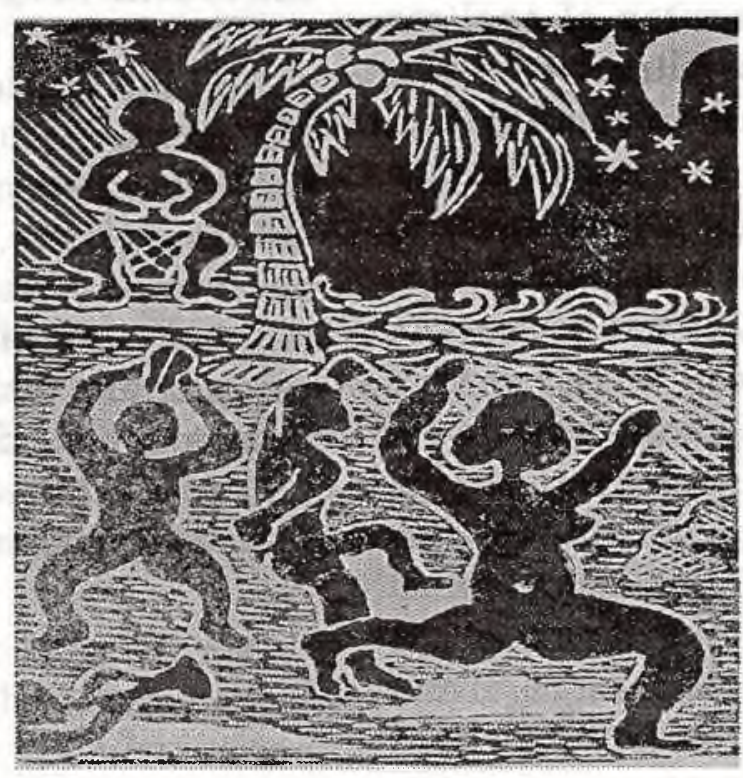
regulación del mercado. canismos corporativos que regulaban las relaciones entre el capital y el trabajo. debilitamiento del concepto estado. bienestar.

- Flexibilidad de la automatización, generando en los países del "tercer mundo", el desarrollo de una industrialización dependiente.

- Traslado de estas industrias hacia países del Sur, buscando las ventajas comparativas (de salarios, materias primas, seguridad social, costos laborales, etc.).

- Abandono de la producción en masa: en la sociedad se empieza a operar una parti- cularización de los gustos, que comienzan a distinguir entre el consumo de elite y el consumo propio de las nuevas clases surgidas del sector servicios.

- Este consumo sofisticado, diferenciado del masivo, supone la existencia de una población educada que se diferencia socialmente por esa cierta exclusividad, creando nuevos estilos de vida, y por lo tanto nuevas formas de exclusión.

- Aparición de la información como un producto de mercado que abre perspectivas ilimitadas a los procesos de reproducción del capital.

- Las exigencias tecnológicas van a presionar cambios en la educación, ya que el trabajador de la producción flexible de finales del siglo, requiere de nuevas habilidades: capacidad de manipular mentalmente modelos, pensamiento conceptual con raciocinio abstracto, comprensión de procesos globales, capacidad de aprender a aprender y de cambiar con el cambio.

- Flexibilización, descentralización y control de la influencia local sobre la escuela, criterios muy en consonancia con los del nuevo paradigma de desarrollo.

- Ampliación de la clase generada en el sector de servicios, lo cual produce el fortalecimiento social y político de las clases medias y genera la atomización del mundo del trabajo, que aún bajo la misma unidad productiva, establece marcada 
diferencia de criterios, intereses y reivindicaciones, ${ }^{3}$

Todos estos elementos se convierten en un reordenamiento y reorientación de la forma de existencia del capitalismo que presiona una reforma de las instituciones sociales en ese mismo orden.

Con relación a la reflexión sobre las profesiones, disciplinas y oficios, conviene señalar que como consecuencia de los desarrollos anteriormente anotados, se asiste al surgimiento de un nuevo modelo de gestión de mano de obra con un cambio fuerte en las características de la fuerza de trabajo, derivadas de una organización basada en la cualificación: esto ha quebrado la "rigidez fordista", en donde se busca ubicar los desarrollos de la tecnología para la posibilidad productiva, generando un nuevo tipo de trabajador, caracterizado por:

- El control de los procesos, es decir va más allá de la vieja tarea fija y previsible. Debe reconocer el proceso global.

- Actitud y disposición hacia las nuevas formas de producción. No solamente disposición a nuevos conocimientos, sino también a toda su actitud de asimilación veloz frente a cualquier aspecto que represente cambio.

- Manipulación mental de modelos.

- Capacidad de hacer equipo, dando pie a una realización de funciones específicas articuladas en la globalidad del proceso que requieren una capacidad individual máxima y del trabajo en equipo.
- Trabajador polivalente. Las características del modelo de desarrollo prevaleciente exigen una persona capaz de cumplir diferentes actividades en el proceso productivo en el cual está inserto, sea este de orden material, intelectual o social. ${ }^{4}$

Lo anterior nos permite señalar que nos encontramos ante un horizonte de acción bastante complejo, y que en la capacidad de acercarnos a dicha complejidad podrán surgir los elementos relacionados con la Pertenencia Social y la Pertenencia Académica de nuestras estructuras curriculares hoy vigentes en la universidad c olombiana.

\section{CAMBIOS EN EL CONOCIMIENTO}

Algunos autores señalan que la humanidad ha vivido cuatro profundas revoluciones productivas: la primera el paso del nomadismo al sedentarismo, dando como resultado la agricultura; la segunda, la utilización de los metales que da la posibilidad de la rueda, la metalurgia y la irrigación; la tercera, la del vapor, que nos lleva a la revolución industrial mecánica, a las máquinas textiles; y la cuarta a la que asistimos hoy, la de la micro electrónica. Cada una de estas revoluciones origina cambios en las maneras de concebir, organizar y pensar la sociedad y el mundo.

En la revolución de la micro electrónica, el conocimiento se convirtió en factor productivo por excelencia, con fenómenos de concentración y acumulación tecnológica basados en la intensidad del conocimiento tecnológico, como señala

3 Mejía, Marco Raúl. Educación y Escuela en el fin del siglo. C.I.N.E.P. Segunda Edición. 1995. Un análisis más detallado de estos planteamientos se encuentran en la línea de trabajo de Marco Raúl Mejía relacionada con la REFUNDACION DE LA ESCUELA Y LA EDUCACION C.I.N.E.P. 
Gorostiaga "Esta Concentración del capital corresponde al carácter de la nueva revolución tecnológica, donde el ciclo de acumulación del capital depende cada vez menos de la intensidad de los recursos naturales y del trabajo, e incluso de la intensidad del capital productivo, para concentrarse en una acumulación tecnológica basada en la INTENSIDAD DEL CONOCIMIENTO. (...). La repercursión de este fenó- meno ha llevado a una desmaterializa ción creciente de la producción donde cada vez se requieren menos materias primas por unidad de producto. Para el caso japonés se ha dado la reducción de un 33 por ciento del uso de materias primas en relación con el producto en los últimos 20 años."

Como consecuencia de lo anterior, la mercancía información asume un valor cada vez más alto y pierde progresivamente su dependencia con respecto al lugar y al tiempo.

P "Los cambios más notorios que nos

E hablan de una nueva época se

D presentan en el saber y el conocimiento y

A su aplicación en la vida cotidiana. Ellos

A son visibles en los cambios tecnológicos

G de la electrónica, la cibernética y la

ingeniería genética, operativizados a través

$G$ de los servicios, la tecnología doméstica e industrial, las computadoras, la bioagricultura y las telecomunicaciones."

Las repercursiones en el campo educativo no se han hecho esperar, hoy adquieren un valor inusitado los MODELOS PEDAGÓGICOS INTEGRADOS que colocan

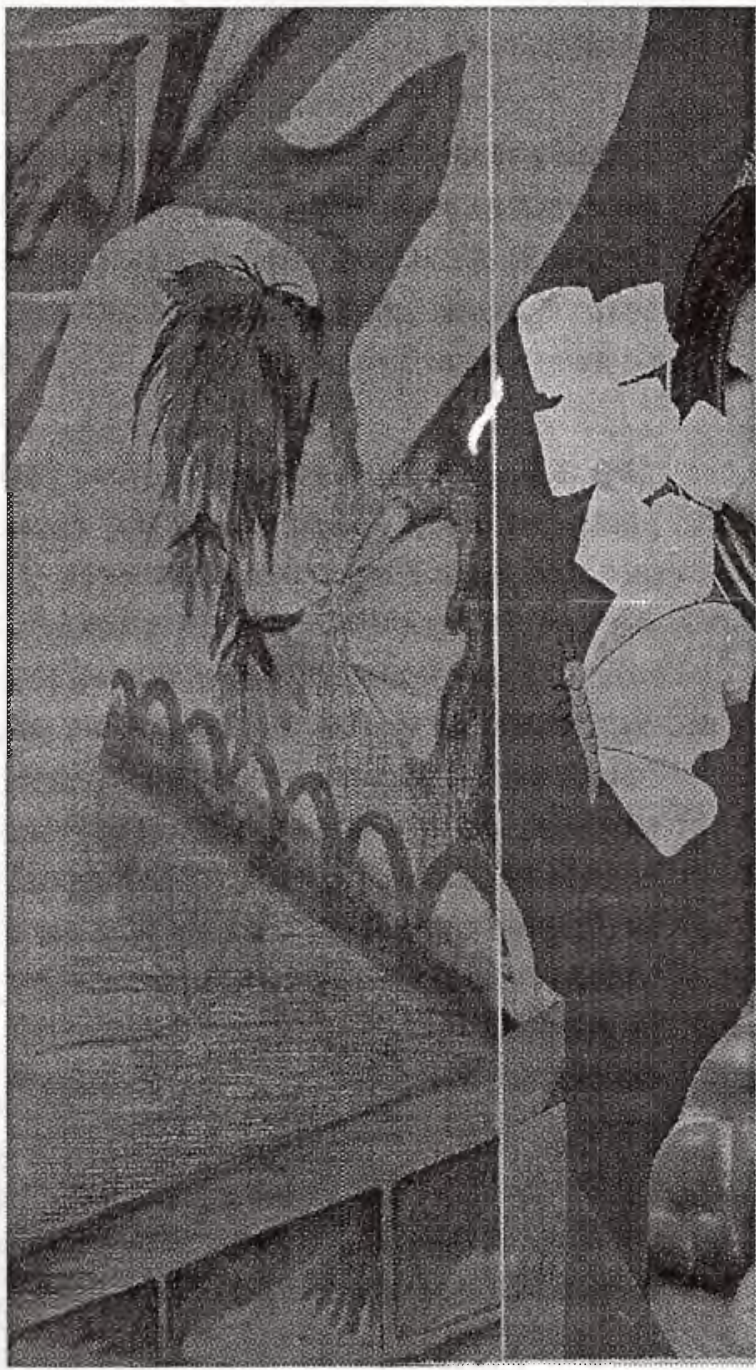

su fuerza en el "conocer como se conoce", "cambiar en medio del cambio", fortalecimiento de los contenidos generativos (intensidad más que cantidad), en la perspectiva de APRENDER A APRENDER, entendido como la acción que permite enlazar pedagógicamente los tres procesos anteriormente descritos.

Igualmente, la velocidad de los cambios también afecta la manera de dar el conocimiento. Se asiste a una competencia educativa, que además de investigación

5 Gorostiaga, Xabier "América Latina frente a los desafios globales"en Cristus. Mayo-junio 1992. pág. 12

6 Mejía. (IBID) pág. 21. 
y enseñanza, exige aceleradamente información actualizada, componente básico de ese conocimiento; un peligro que se evidencia en los procesos curriculares es el de "intoxicar de información" a la sociedad descuidando los fundamentos y los procesos básicos.

\section{UNA MIRADA A LA CULTURA CURRICULAR ACTUAL EN LA EDUCACIÓN SUPERIOR EN COLOMBIA}

Como premisa inicial, es importante señalar que la preocupación cultural que se genera en torno al problema curricular. en Colombia, es un indicativo válido para afirmar la condición de objeto de saber, de objeto de investigación que se le ha otorgado al curriculum; se advierte una percepción diferente a la tradicional, que consideraba el currículum como algo ya definido, determinado que solamente se movía en la racionalidad de la ejecución. en lo operativo, en lo mecánico. Obviamente, dicha preocupación está en gestación: todavía existe una tendencia prevaleciente que le da o le atribuye un carácter algorítmico y acrítico, reafirmando la dominación e imposición cultural.

Es conveniente precisar que inicialmente se presentarán algunas expresiones de la naturaleza actual del proceso curricular, que permita en la parte complementaria socializar una alternativa curricular defendida y argumentada desde la integración del conocimiento (académicocotidiano-socialización) en la perspectiva de contribuir a la ampliación del horizonte de acción, hacia la construcción de currículos con arraigo social y pertinencia académica.

Al intentar diagnosticar la situación relacionada con el proceso curricular, es de obligada referencia, afirmar la carencia de proyectos educativos políticos y culturales, que le den imbricación a esta dinámica, es la impronta establecida como común denominador, en otras palabras, muchas acciones curriculares se adelantan. desprendidas, aisladas, de un marco o política institucional, que recoja el tipo de hombre que se quiere formar, la sociedad que se desea lograr, la identidad cultural que se aspira consolidar o recuperar, las metas por las cuales hay que trabajar. No existe una continuidad de propósitos, y en la mayoría de los países, se avanza por pálpitos, por impulsos desordenados que tienen una vida fugaz, muy condicionadas a la duración de las administraciones políticas de turno.

Un aspecto importante a considerar, tiene que ver con la influencia marcada en nuestros países, de la denomida Tecnología Educativa (básicamente a través del diseño instruccional), en la que se concibe que la acción curricular únicamente hace referencia a la formulación de objetivos. selección de contenidos, definición de actividades tanto de alumnos como de profesores, rigidez en la evaluación, etc. como producto de la denominada "Taylorización del proceso curricular", hoy se evidencia y se acepta sin mayores reparos la existencia de una clasificación fuerte (siguiendo a Bernstein) entre los que producen cultura, los que transmiten cultura y los que adquieren cultura. El docente es visto como un apéndice del proceso curricular y no como un elemento en, desde y dentro del conocimiento.

En la actual estructura curricular del sistema educativo, prevalece la cultura académica, de colección, enciclopédica, atomizada a través de asignaturas n micropoderes, que impiden cualquier pretensión de formación integral, toda vez que la cultura comunitaria, cotidiana, de socialización, posee poca, por no afirmar 
ninguna importancia. Al privilegiar la cultura disciplinaria como el dispositivo por excelencia a transmitir, se excluyen otras formas de saber, arrojando como resultado una educación descontextualizada que reafirma el proceso de fraccionamiento que se adelanta a través de la educación. No existe una educación

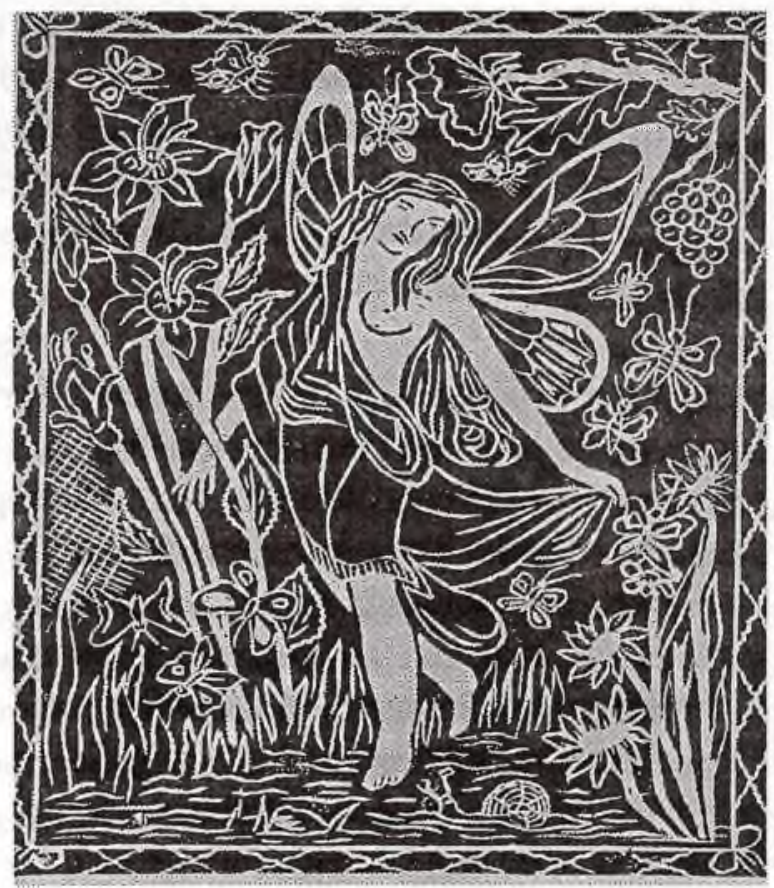

integral ni integradora que responda a los proyectos culturales básicos.

Son estructuras cerradas, con límites y fronteras definidas, selectivas y discriminatorias, que ponen de manifiesto mecanismos de acomodación, rutinización y ausencia de innovación y cambio. Es interesante reflexionar sobre los criteros y fundamentos que soportan la clasificación fuerte entre saber académico y no académico, ya que lo académico no agota el concepto de integralidad, hay autores que afirman que lo debilita, "términos como el consenso, la representatividad, lo unitario, la formación integral, servirán como subterfugio para ocultar el carácter reproductor de una sociedad desigual que se desea reproducir en el currículum a través de la imposición de los códigos culturales dominantes."7

Como resultado natural de una acción de clasificación fuerte, el proceso pedagógico se sustenta sobre la autoridad incuestionable del maestro; por lo general, la acción metodológica descansa excesivamente en la exposición oral tradicional, se evidencia un énfasis marcado en la enseñanza dejando a un lado la problemática inherente al aprendizaje; las expectativas y derechos de los alumnos son ignorados, porque se maneja un concepto genérico de "estudiante" que impide tener algún conocimiento específico o distintivo del mismo. Los estudiantes "todos" son iguales. Se evidencia una fuerte enmarcación en la relación docente-estudiante, los roles están definidos y las relaciones se mueven dentro de un contexto donde el maestro tiene el máximo de control y poder, la verticalidad en la relación profesor-alumno es lo que prevalece significativamente.

Pretender desconocer la profesionalización y solvencia académica del personal docente, es un argumento que no hace justicia con la realidad, no obstante, la actual estructura curricular hegemónica propicia un desempeño aislado y fraccionado; el individualismo y la

7 Magenzo, Abraham. Análisis Crítico conceptual de la concepción curricular centrada en las disciplinas de estudio. P.I.I.E. Santiago de Chile 1991. pág. 60. 
competencia personal son fomentados por las estructuras curriculares rígidas y jerarquizadas. No existen ejes de integración, cuando la acción docente se mueve en ambientes finamente diagonalizados por la concurrencia de micropoderes.

La interdisciplinariedad, la transdisciplinariedad son ideales por conseguir, que no se ven favorecidos, pues cuando se legitima la dispersión y el trabajo desintegrado, se debilita la intención de constituir verdaderas y auténticas comunidades académicas, el trabajo en grupo o colectivo no es la característica esencial de la labor pedagógica.

En consecuencia, resulta válido analizar hasta dónde es posible avanzar en un cambio actitudinal, en un compromiso fuerte con los propósitos institucionales, si se siguen manteniendo e institucionalizando estas formas de desempeño y labor profesional, que debilitan permanentemente la relación entre docentes y terminan contribuyendo a un "proceso de formación" en donde no se es consciente de la intencionalidad del mismo.

Si se pretende fomentar y propiciar la creación de comunidades académicas, investigativas y científicas, necesariamente se tienen que intervenir las actuales manifestaciones de poder, a través de las estructuras curriculares agregadas o academizadas.

Es importante señalar que la investigación no es un elemento central en la dinámica curricular en los sistemas educativos latinoamericanos, colocando en una posición cuestionable las alusionés a la calidad y excelencia del proceso mismo. Afirmar que el desarrollo, impulso y consolidación de una estructura investigativa coherente con los propósitos regionales, nacionales, institucionales en mate- ria educativa, debe ser asumido como tarea central en la reorientación del proceso educativo, es reconocer crítica y objetivamente la ausencia y carencia de la misma.

Para ilustrar la anterior afirmación, es necesario traer a colación la situación de Colombia en materia investigativa, según estudio de Colciencias (organismo oficial que administra la política de investigación) la escasa investigación que se realiza en el país se concentra en siete universidades (cuatro estatales y tres particulares); es decir, del total de instituciones de Educación Superior solamente se puede afirmar que $2.5 \%$ asumen la investigación con un carácter básico en su funcionamiento.

La evaluación como proceso inherente a todo desarrollo educativo, ha sido marginada en el proceso curricular de Latinoamérica, quizá por influencia heteronómica existente (la fuerza de la normatividad): se ha caracterizado por ser un proceso intermitente, por saltos, por intuiciones, que propician un ambiente favorable a la improvisación y el eventualismo.

La cultura evaluativa no ha podido ser introyectada como un fenómeno necesario y connatural al quehacer académico; se considera que se debe evaluar porque "algo anda mal", el carácter punitivo que acompaña al proceso evaluativo, no ha permitido entender la incidencia e importancia del mismo.

De lo anterior se deduce que hoy en Colombia, a través de su cultura curricular, no ha sido abocada la revolución del conocimiento y mucho menos las exigencias derivadas de un modelo de desarrollo que genera procesos de reorganización, reconstrucción y recomposición de las instituciones sociales. 
4. CARACTERÍSTICAS BÁSICAS PARA EL ANÁLISIS DE LA FORMACIÓN TECNOLÓGICA, CIENTÍFICA EN LAS DIFERENTES ÁREAS DEL CONOCIMIENTO EN LA EDUCACIÓN SUPERIOR.

Es importante invalidar de una vez por todas que el criterio por excelencia para definir los procesos de formación técnica, tecnológica y profesional, sea el tiempo o duración de la carrera ${ }^{8}$ toda vez que existen otros referentes que tienen que ver con la naturaleza misma del conocimiento. en esa dirección resulta muy oportuno consignar lo

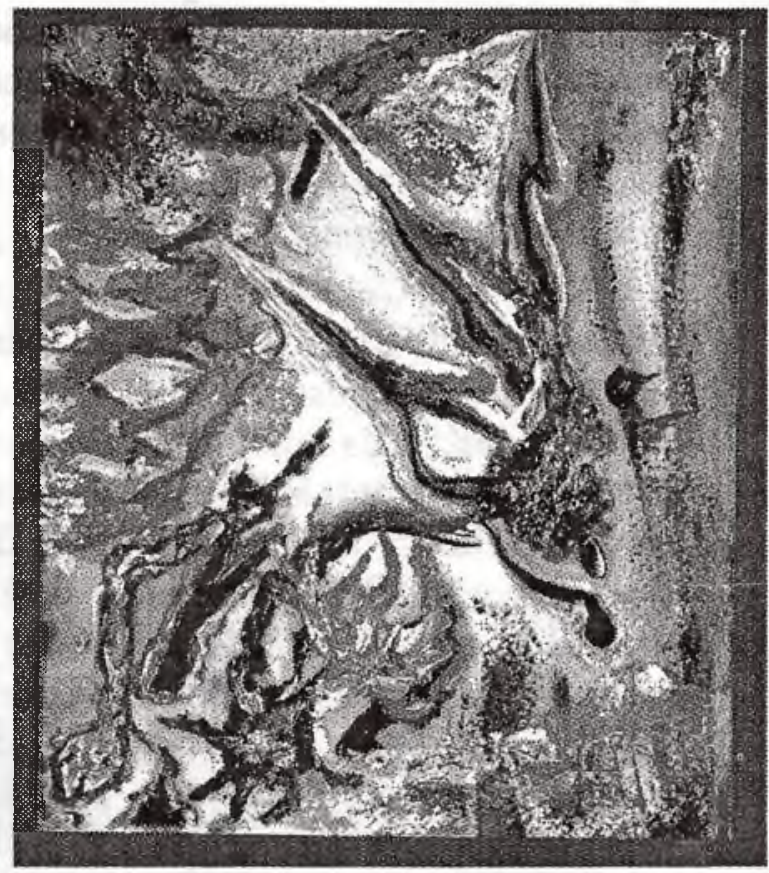
planteado en el documento LAS PROFESIONES HOY Y SUS RELA CIONES CON EL TRABAJO Y EL EMPLEO ${ }^{9}$ que señala "como criterio para clasificar las profesiones, la aplicación de las ciencias, y se vale de cuatro expresiones literales así: las profesiones intelectuales o académicas FCI, porque su fundamento es, casi exclusivo, científico e investigativo. Las profesiones intelectuales prácticas ( $\mathrm{FCl} / \mathrm{P})$, porque del sólido fundamento científico e E investigativo, depende la calidad del D ejercicio profesional. Las profesiones tecnológicas. (P/FCI), porque estando ante todo, orientadas a la práctica, consultan el fundamento científico, investigativo de lo que se hace; las profesiones técnicas ( $P$ ). que se ocupan de la práctica, sin mayor o aún ninguna atención a la razón científica de lo que se opera o practica.

Sin embargo, estos elementos no han permeado la discusión, acción y decisión de la dinámica universitaria, y hoy vemos con asombro y preocupación que la "metástasis programática en la Educación Superior" (en diciembre de 1992, fecha de expedición de la Ley 30, existían 2.219 programas en las diferentes modalidades, a la fecha, según las últimas estadísticas del ICFES, la cifra va en 4.279 programas, un incremento del $96 \%$-igual al que tuvo la educación superior en las últimas tres décadas), en donde la confusión, diversidad, ambigüedad, pero fundamentalmente la irracionalidad académica, es el aval fundamental de la situación presentada.

Si retomamos lo planteado en la parte inicial de este trabajo, resulta aún más penosa la situación de nuestra educación universitaria; no tenemos como referencia la producción de conocimiento, ni el

8 Quizá este fue uno de los aspectos más cuestionables que marcó la dinámica de las instituciones de educación superior durante la vigencia de la Ley $80 / 80$.

9 Documento elaborado por ASCUN, presentado en el Encuentro Internacional sobre Crisis Mundial de la Educación Superior. Bogotá, abril de 1986. 
conocimiento mismo, mucho menos el desarrollo científico y tecnológico actual, pero avanzamos en un proceso de proliferación y devaluación académica, que probablemente construirán una nueva "frustración nacional" que demorará la posible incursión en el campo natural donde se mueven las comunidades académicas y científicas contemporáneas de nuestra Universidad.

Si de dilemas se trata, es preciso abordar esta situación con el mayor sentido de responsabilidad, so pena, de asumir una actitud de complicidad con el proceso de "engañamiento social" que hoy se quiere consolidar vía educación superior ${ }^{10}$.

Sin temor a equivocación, se podría colegir que las características. los fundamentos, las exigencias, y los determinantes del saber científico, del saber técnico, del saber tecnológico, no han sido tratados como referentes sustantivos en la formación de los profesionales en las diferentes áreas del conocimiento, lo cual ha originado una confusión que apenas pretende ser aclarada por instituciones que orientan su desarrollo basadas en una rentabilidad académica y social, pero que no han sido atendidas por las instituciones que solo se orientan por la rentabilidad económica, que para el caso colombiano, son la mayoría.

\section{LINEAMIENTOS CURRICULARES BÁSICOS}

Es preciso señalar que uno de los referentes fundamentales a tener en cuenta es el conocimiento, su producción. su aplicación. su estructuración en procesos científicos y tecnológicos como se planteó en los primeros tópicos de este documento, sin embargo, con la intención más de informar que de analizar detenidamente, se reseñan algunos criterios que han sido sistematizados para la clasificación de las profesiones ${ }^{11}$, que si bien alguno de ellos pueden rescatarse, es necesario una reconstrucción permanente desde el carácter interdisciplinario, integral, investigativo y pedagógico mediatizados por la construcción curricular asumida como una acción eminentemente creativa.

Importante señalar que las disciplinas hacen referencia al cuerpo teórico que identifica a un saber constituido por conceptos y leyes de carácter general que son reconocidos como tales por la tradición científica en ese campo; desde esta perspectiva se le asigna un relativo carácter paradigmático. En consecuencia, la interdisciplinariedad como actitud mental ${ }^{12}$, debe asumirse como un proceso y una filosofía de trabajo que se pone en acción a la hora de enfrentarse a los problenas y cuestiones que si preocupan en cada sociedad ${ }^{13}$.

Esta situación ha originado que muchas entidades empleadoras, fijen en el perfil de requisistos que debe reunir el candidato, que sea profesional de X universidad. En este sentido, se abre paso el concepto de legitimidad y acreditación social, como un nuevo mecanismo de exclusión.

1 Entre los cuales se destacan: criterio cronológico; criterio del campo científico sustentante; criterio fundado en la aplicación de las ciencias; criterio por la raigambre con la naturaleza activa del hombre; criterio fundado en los cambios de identidad de las profesiones; criterio conforme con la jerarquía investigativa de las profesiones; criterio fundado en las formas del ejercicio profesional; criterio basado en la prestación colectiva de servicios profesionales; criterio para discen s. sobre el control estatal sobre las profesiones. Tomado ASCUN: II Encuentro Nacional de Autoevaluación. Paipa, junio /88. 1995.

12 ANDER, Ezequiel Eqq. La interdisciplinariedad en Educación. Ediciones Pedagógicas, Argentina, 1995.

13 TORRES, Jurgo. Globalizacion e Interdisciplinariedad: ElCurriculum integrado. Ediciones Morata. Madrid, 1995 
La interdisciplinariedad se acostumbra a asociar también con el desarrollo de ciertos rasgos de la personalidad, tales como la flexibilidad, confianza, paciencia, intuición, pensamiento divergente, capacidad de adaptación, sensibilidad hacia las demás personas, aceptación de riesgos, aprender a moverse en la diversidad, a aceptar nuevos roles, etc.

En esta fundamentación es en la que se basa la propuesta curricular sobre Núcleos Temáticos y Problemáticos, Bloques Programáticos y Proyectos Puntuales, que se viene trabajando como referente específico hacia la modernización de las estructuras curriculares en la educaciónsuperior colombiana $^{14}$.

"La interdisciplina riedad es un objetivo nunca alcanzado por completo y de ahí que deba ser permanentemente buscado. No solo es un planteamiento teórico, es ante todo una práctica. Su perfectabilidad se lleva a cabo en la práctica, en la medida en que se desarrollan experiencias reales de trabajo en equipo, se ejercitan sus posibilidades, problemas y limitaciones. Es una condición necesaria para la investigación y la creación de modelos más explicativos de esta sociedad tan compleja y difícil de abarcar ${ }^{115}$.

Importante destacar que la interdisciplinariedad será la disciplina del próximo siglo.

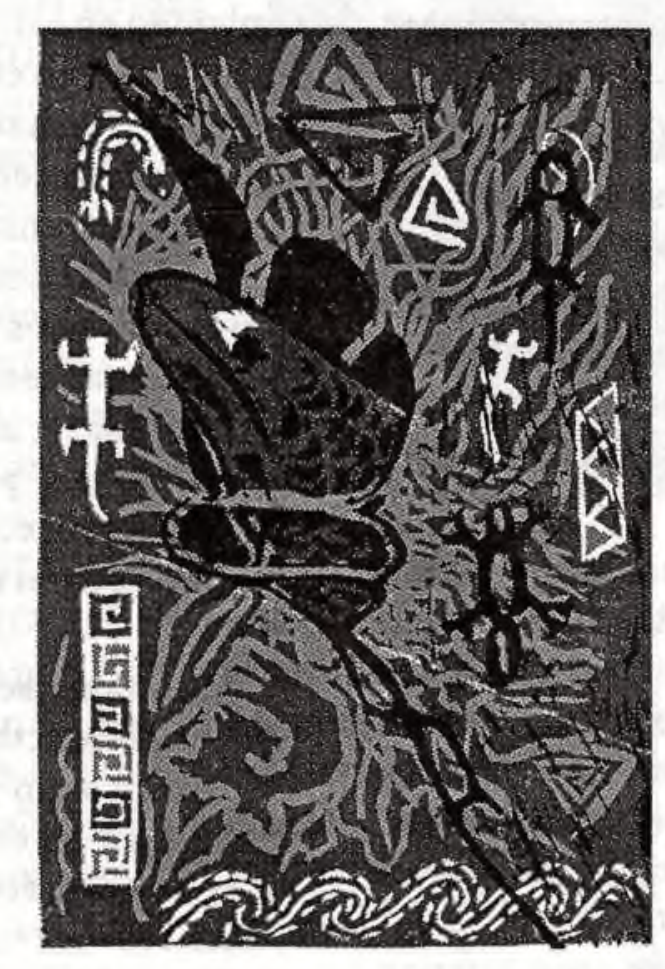

En cuanto al criterio de integración $\mathrm{Cu}$ rricular. obedece a la necesidad de crear, desarrollar y consolidar comunidades académicas tanto en el campo de las disciplinas como en el de las interdisciplinas. La integración, considerada como la relación de los elementos que conforman un todo, no pretende ser tomada aquí simplemente como la yuxtaposición de partes para dar unidad a una estructura, porque el criterio no alude a la importancia de los elementos, sino a la interrelación de ellos como lo fundamental, en cuanto es ese vínculo orgánico el que genera la dinámica y propicia los procesos curriculares ${ }^{16}$.

Si bien se puede afirmar que el concepto de integración habita en muchos discursos sobre la educación, no menos cierto es, que en la práctica concreta la cultura de la individualidad, el atomismo.

14 Línea de investigación, apoyada inici:i*nente por COLCIENCIAS e ICFES y que continúa su desarrollo con el apoyo de ICFES y la Universidad Surcolombiana, dirigida por el autor de este documento.

15 TORRES, Turjo. (IBID) PAG. 69.

16 ISAZA VÉLEZ, Francisco Javier. La Reforma Curricular en Ciencias Sociales: Una hipótesis que se demuestra con producción académica. Univ. Distrital Francisco José de Caldas. Bogotá, 1995. 
el aislamiento, la insularidad, son fenómenos prevalecientes actualmente.

Las formas de convivencia social no se dan naturalmente, son creadas y construídas por el hombre. Por no ser un hecho natural, la convivencia social puede ser aprendida y puede ser enseñada ${ }^{17}$.

Razón por la cual si es posible transformar nuestra cultura curricular procurando transformar el paradigma de la imposición por el paradigma de cooperación, concertación y negociación, lo cual exigirá que enfrentemos decididamente, entre otros, la integración de los siguientes espacios culturales:

- Integración de la escuela a la vida. No se educa para la vida, sino que debe entenderse que la educación es vida.

- Integración del sector educativo con los sectores políticos, económicos, industriales, comerciales, culturales del país. Garantizar un diálogo permanente de los diversos sectores en procura de determinar las necesidades reales del país y los compromisos concretos que se asuman para solucionarlas.

- Integración de la cultura académica, disciplinaria, enciclopédica, con la cultura de la cotidianidad y con la cultura emanada de los escenarios primarios de socialización (familia, barrio, el campo deportivo, la organización comunal, etc.)
- Integración de la educación, la ciencia y la tecnología, toda vez que "el futuro de Colombia va a estar profunda y directamente relacionado con la capacidad que los colombianos tengamos de organizar la educación: la hija de la educación: la ciencia; la hija de la ciencia; la tecnología. Sin la menor duda este estrelazamiento será uno de los ejes principales del futuro de nuestro país en el siglo $\mathrm{XXI}^{118}$.

- Integración de las disciplinas, entendida como el reto mediante el cual se garantiza la "concurrencia simultánea y/o sucesiva de saberes, sobre un mismo problema, proyecto o área temática"19.

- Integración entre los diferentes niveles educativos y modalidades. No puede seguir pensándose el "sistema" educativo como una yuxtaposición de intencionalidad y micropoderes.

En consecuencia, se puede deducir que la integración curricular es un reto por enfrentar $y$ un proceso por construir.

En lo relacionado con la investigación. es preciso señalar que está directamente relacionado con el "cultivo de la capacidad de asombro, de duda y de sospecha de todos y cada uno de los procesos y actores de la acción formativa". Dilucidar la intencionalidad de la labor investigativa y crear al interior de los diversos escenarios sociales las condiciones básicas para la construcción, adaptación, difusión y sistematización de conocimientos, brindarán "pistas" definitivas en el estudio de la problemática de las profesiones.

${ }_{17}$ Fundación Social "Siete Aprendizajes Básicos para la Educación en la Convivencia Social" en educando para hacer posibles la vida y la felicidad. Bogotá, 1993.

${ }_{18}$ Llinas, Rodolfo. Revolución positiva sin precedentes. Misión Ciencia Educación y Desarrollo. Documento de presentación. Febrero 1994.

19 Yunis, Emilio. "Una visión interdisciplinaria de la Ciencia" en Simposio Internacional sobre Investigación Científica. Bogotá. 1993. 


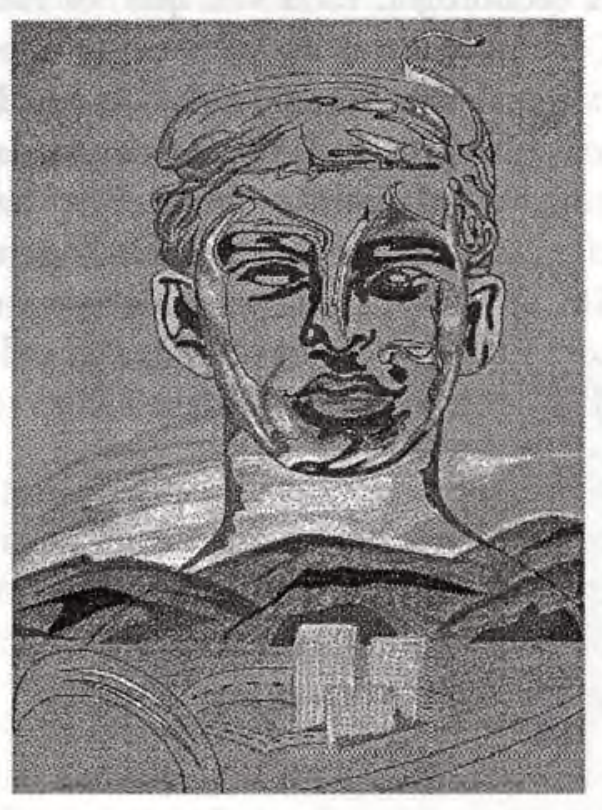

Aquella intención de otorgar el máximo de credibilidad a los modelos importados, ha fortalecido la necesidad de crear nuestras propias respuestas a los cuestionamientos formulados; la actitud pasiva y permisiva de nuestras instituciones en el campo investigativo, debe ser recordada como algo pretérito; se convierte la necesidad de integrar equipos investigativos como el dispositivo ético y profesional que facilitará los procesos de pertenencia social y pertinencia académica de toda construcción curricular.

Se asume que el proceso curricular es una acción eminentemente investigativa, razón por la cual los criterios, procesos, estrategias, contenidos, etc; deben ser producidos y construidos en las entrañas de la práctica formativa profesional de la misma.
El carácter investigativo de una estructura curricular, no se alcanza con la introducción de cursos de investigación en su plan de estudios, al considerarse la actividad investigativa como elemento fundamental de formación, como principio de conocimiento y de la práctica, posibilita la recreación y reconstitución del proceso formativo de cara a u ua realidad social nueva que desborde los marcos de cambio institucionalizados, con el fin de transformar sus estructuras, métodos y programas, lo mismo que el proceso básico de enseñanza-aprendizaje, y logren en la práctica el anhelado rasgo de pertenencia social y pertinencia académica del proceso desarrollado.

En lo correspondiente a la dimensión pedagógica es conveniente destacar la necesidad de "desplazarnos" de un modelo pedagógico tradicional, clásico, agregado a la construcción de un modelo pedagógico integrado ${ }^{20}$, que nos permita construir una nueva mirada de la problemática de la formación profesional y disciplinaria, en donde se recoja sustantivamente los avances de la revolución de la informática y la implicación de dichos avances en el campo social, investigativo, cultural, científico y disciplinario.

Es preciso enfatizar que "emergen unos saberes nuevos que son formas reflexivas propias de tiempo de cambio y que muestran la perspectiva del proceso. Allí están elmentos como: aprender a aprender, conocer como se conoce, cambiar en medio del cambio, procesos que adquieren centralidad en la acción educativa misma. ${ }^{21}$

${ }^{20}$ Un análisis detenido del concepı Modelo Pedagógico, puede encontrarse en los trabajos de Rafael Flores. Pedagogía de la Producción del Conocimiento; Mario Díaz: La Construcción Social del Discurso Pedagógico; Nelson López; La Reestructuración Curricular de la Educación Superior. Hacia la Integración del Saber.

${ }^{21}$ MEJĹA, Marco Raúl. Reconstruyendo la transformación social. Movimientos sociales y educación popular. Mesa Redonda. Magisterio No. 43. Agosto 96. 
La flexibilidad curricular como consecuencia de procesos de pertenencia social y pertinencia académica, debe ser entendida como un factor determinante en una posible acción clasificatoria de las profesiones, toda vez, que su carácter dinámico impide los reduccionismos y abre el espacio hacia la incertidumbre creativa y constructiva, básica en los actuales momentos por los que atraviesa la educación colombiana.

\section{REFLEXIONES FINALES}

Reconocer la hegemonía de un modelo de desarrollo económico al final del siglo, y su influencia y exigencia en la transformación y recomposición de las organizaciones y de las instituciones sociales, no puede entenderse como la salida facilista de adecuar la dinámica de la universidad a las exigencias del mercado, tampoco ignorarlo; pero sí recuperar el sentido y la acción que han caracterizado la existencia de la institución universitaria (notas fundamentales). Se hace necesario, instalar la reflexión sobre la universidad. en un campo en recomposición y en ese sentido, es lucha entre visiones, que tratan de realizar acciones desde las concepciones que muestran que no solo es necesaria otra universidad, sino que se hace urgente la transformación de sus procesos.

La propuesta de cambio de la "escuela" derivada del poder económico, funda- mentada en el conocimiento, no puede ignorar que la universidad se mueve en el contexto de la producción del conocimiento, por ello, la tarea de reinvención de la teoría crítica y de la acción transformadora en el campo de la educación, pasa por la capacidad de entender las transformaciones capitalistas del final del siglo y desde allí instaurar los nuevos elementos que avancen a construír una educación que realmente garantice la equidad, la justicia, la alteridad, la tolerancia, como paradigmas de acción que guien y orienten la refundación de la escuela y obviamente la transformación de la sociedad.

Es en este contexto, que considero se debe instalar la reflexión sobre los dilemas surgidos entre las profesiones, disciplinas y oficios, procurando no minimizar la complejidad del fenómeno estudiado, pero sí dar oportunidad de desandar lo andado. es decir, intentar abandonar la certeza desde donde hablamos y enrutarnos en el camino de la incertidumbre, la sospecha, la duda. Por ello, el asumir el proceso curricular como un proceso investigativo debe entenderse como la explicitación de un reto por enfrentar y un proceso por construir, es en palabras de Vattimo, "ser capaz de pasar de un pensamiento fuerte a un pensamiento débil, con todas las consecuencias que esto implica en términos de despojo y de abandono de las verdades absolutos que tuvimos para poder iniciar la búsqueda"22.

\footnotetext{
I2 Vattimo, Gianni, y Pier Aldo Rovatti. El pensamiento Débil Cátedra. Madrid. 1990
} 